\title{
Analysis of the influence of ultrahigh pressure on wellbore Fluid properties_-Take Well ST1 in north Sichuan as an example
}

\author{
Liu Qilin ${ }^{1, a^{*}}$ \\ ${ }^{1}$ CNPC Southwest Oil and gas field Branch Sichuan Northwest Gas mine, Mianyang, China
}

\begin{abstract}
According to the research on wellbore pressure temperature prediction of ultra-high pressure gas Wells, the influence of ultra-high pressure on wellbore fluid physical property parameters cannot be ignored, the component model is adopted to calculate wellbore fluid PVT physical property, and the multi-phase flow model is modified to accurately predict wellbore pressure temperature distribution. For the prediction of gas deviation factor of well flow and gas viscosity of well flow, the component model has a high precision. By comparing with the prediction results of 8 black oil model methods, the pressure has a great influence on the black oil model. When the pressure is equal to $100 \mathrm{MPa}$, the deviation value between the predicted results of Gopal method and Dranchuk-Abu-Kassem method and the component model is greater than 0.1, which can no longer guarantee the accuracy of gas deviation factor and gas viscosity prediction. Therefore, it is recommended to use the component model to predict the deviation factor and gas viscosity of gas well flow.
\end{abstract}

\section{Introduction}

In recent years, CNPC Southwest Oil and Gas Field Company has made great breakthroughs in gas exploration of the qixia ultrahigh pressure gas reservoir in the Lower Permian, which is expected to become another important exploration and development area in Sichuan Basin. At the same time, the theoretical research on the development of uHP gas Wells has also made great progress, and scholars on the characteristics of China's uHP gas wells test production $^{[1-3]}$, capacity analysis ${ }^{[4-6]}$, and upporting technology ${ }^{[7-9]}$, a great deal of research has been carried out and fruitful results have been achieved. However, there are few researches on the wellbore flow characteristics of uHP gas Wells at present. The wellbore fluid property is the bridge between the surface production of gas well and the formation inflow, and the basis of analyzing the gas well productivity and gas reservoir reserves, the research value of which is very important. Therefore, the author starts from the fluid physical characteristics of ST1, a typical uHP gas well in Sichuan Basin of China, and systematically analyzes the comprehensive influence of uHP gas well uHP on gas deviation factor and gas viscosity.

In the study and calculation of gas well wellbore multiphase pipe flow, the pressure gradient equation of single-phase fluid one-dimensional stable pipe flow is generally based on the following equation:

$$
\frac{d p}{d z}=\rho_{\mathrm{m}} g \sin \theta+f_{\mathrm{m}} \frac{\rho_{\mathrm{m}} v_{\mathrm{m}}^{2}}{2 D}+\rho_{\mathrm{m}} v_{\mathrm{m}} \frac{d v_{\mathrm{m}}}{d z}
$$

Where: $\rho_{\mathrm{m}}$ is density of mixture; $v_{\mathrm{m}}$ is The speed of the mixture; $f_{\mathrm{m}}$ is The friction of the mixture.

These three parameters are closely related to the physical properties of multiphase flow mixtures. When analyzing the characteristics of gas wellbore multiphase flow, it is necessary to predict the physical characteristics of gas wellbore fluid PVT. For atmospheric gas Wells, the traditional black oil model is often used. For uHP gas Wells, the traditional black oil model is difficult to accurately calculate the wellbore fluid PVT physical characteristics. Therefore, based on double agent 1 well fluid composition data, as shown in table 1 , the introduction of PVT component model gas well fluid physical properties, such as gas viscosity, density, in order to accurately describe the change law of ultrahigh pressure the wellbore fluid PVT, and predict wellbore pressure - the depth, temperature - deep relationship, provide the basis for the ultrahigh pressure the wellbore multiphase flow characteristics analysis.

Table 1 Fluid component analysis data of well ST11

\begin{tabular}{|c|c|c|c|c|c|c|c|c|c|}
\hline \multicolumn{6}{|c|}{ Composition (\%) } & \multirow{2}{*}{$\begin{array}{l}\mathrm{H}_{2} \mathrm{~S} \text { content } \\
\left(\mathrm{g} / \mathrm{m}^{3}\right)\end{array}$} & \multirow{2}{*}{$\begin{array}{l}\text { Critical } \\
\text { temperature }(\mathrm{K})\end{array}$} & \multirow{2}{*}{$\begin{array}{l}\text { Critical } \\
\text { pressure (MPa) }\end{array}$} & \multirow[b]{2}{*}{ Relative density } \\
\hline methane & ethane & Carbon dioxide & helium & hydrogen & nitrogen & & & & \\
\hline 96.09 & 0.14 & 3.05 & 0.027 & 0.001 & 0.69 & 0.226 & 193.7 & 4.68 & 0.5867 \\
\hline
\end{tabular}




\section{Influence of ultra-high pressure on gas deviation factor model}

\subsection{Model deduction,}

There are many calculation methods for deviation coefficient $\mathrm{Z}$ of natural gas. Black oil model and component model are used to compare and analyze the influence of ultra-high pressure on gas deviation factor model.

The component model of phase balance calculation of oil and gas reservoir fluid can obtain the overall properties of the fluid and the properties of each component. Typical calculations of phase equilibrium conditions can be divided into two categories: first, a set of composition and property data under given temperature and pressure conditions is required; second, for known composition and pressure or temperature conditions, look for temperature or pressure in other saturated states. Most phase equilibrium calculations are limited to two phases, i.e. vapor-liquid equilibrium. Each component has the same key fugacity in all phases, but the equilibrium condition determined by the number of phases may also be ignored.

You flash 1 mole of the mixture at pressure $\mathrm{P}$ and temperature T into $n^{\mathrm{L}}$ mole of liquid and $n^{\mathrm{V}}$ mole of gas. The total material balance of the system is:

$$
n^{L}+n^{V}=1
$$

The material balance of each component $\mathrm{I}$ is:

$$
z_{i}=x_{i} n^{L}+y_{i} n^{V}, i=1,2, \ldots, N
$$

Where $\mathrm{z}_{\mathrm{i}}, \mathrm{x}_{\mathrm{i}}$ and $\mathrm{y}_{\mathrm{i}}$ are the mole fractions of component $\mathrm{I}$ in the mixture of liquid and gas.

$$
\sum_{i=1}^{N} x_{i}=\sum_{i=1}^{N} y_{i}=1
$$

Where $\mathrm{N}$ is the total group fraction of the system. At equilibrium, the fugacity of any component $\mathrm{I}$ in the gas phase is equal to the fugacity of the liquid phase. Equality of fugacity is expressed by equilibrium ratio $K_{i}$,

$$
K_{i}=y_{i} / x_{i}, i=1,2, \ldots, N
$$

Substitute formula (5) into formula (3) and get:

$$
\begin{aligned}
& x_{i}=\frac{z_{i}}{1+\left(K_{i}-1\right) n^{V}} \\
& y_{i}=\frac{K_{i} z_{i}}{1+\left(K_{i}-1\right) n^{V}}
\end{aligned}
$$

When $\mathrm{K}_{\mathrm{i}}$ is known, substitute the above two equations into (4) to determine the value of the $n^{\mathrm{L}}$ and $n^{\mathrm{V}}$. The results of this equation need to be solved by iterative method, and the rachford-Rice equation is preferred, the value decreases monotonically with increasing $n^{\mathrm{V}}$ :

$$
f\left(n^{V}\right)=\sum_{i=1}^{N}(y i-x i)=\sum_{i=1}^{N} \frac{z_{i}\left(K_{i-1}\right)}{1+\left(K_{i}-1\right) n^{V}}=0
$$

\subsection{Computed result}

The above formula can be used to determine the state of the fluid at a given temperature and pressure. If unacceptable result is found through iteration which is $n^{\mathrm{V}}>1$ or $n^{\mathrm{V}}<0$, then you can think of the mixture as either gas or liquid.

The fluid component data of ST1 were input into the PVT component model to calculate the gas deviation factor of the well flow. By comparing the prediction results of eight black oil model methods, including Gopal method, Carlile- Gillett method, Cranmer method, HallYarbough method, Dranchuk-Purvis-Robinson method, Dranchuk-Abu-Kassem method, Hankins-Tomas-Phillips method and Sarem method, the influence of ultra-high pressure on the gas deviation factor of the well flow was analyzed. The results are shown in FIG. 1 and FIG. 2.

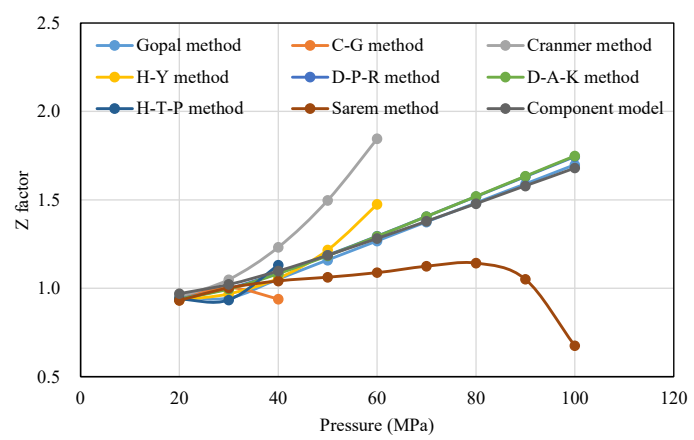

Fig1. Comparison of gas deviation factor $\left(140^{\circ} \mathrm{C}\right)$

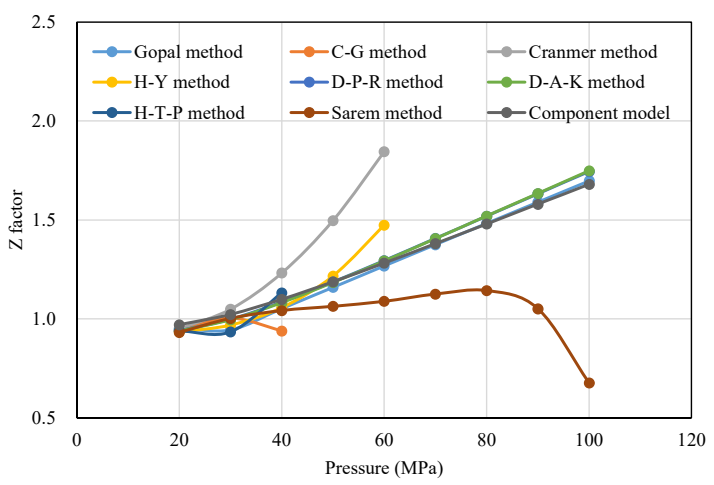

Fig2. Comparison of gas deviation factors $\left(150^{\circ} \mathrm{C}\right)$

Combined with PVT phase analysis of gas reservoir fluids in ST1, the curve of flow deviation coefficient change with pressure in double exploration well 1 was compared with the model curve established in this paper (Figure 3, Figure 4). It can be seen from the comparison that the component model has high accuracy and can effectively predict the gas deviation factor of ST1.

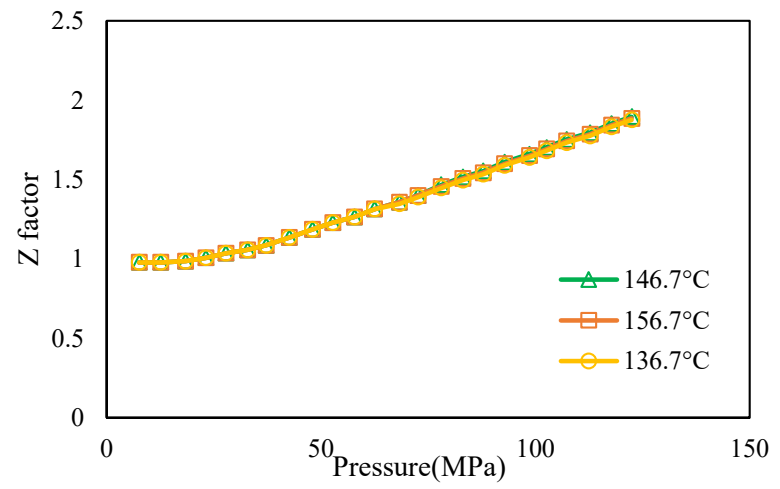

Fig3. Variation curve of deviation factor with pressure (taken from The Natural Gas Reservoir Fluid Inspection Report of ST1) 


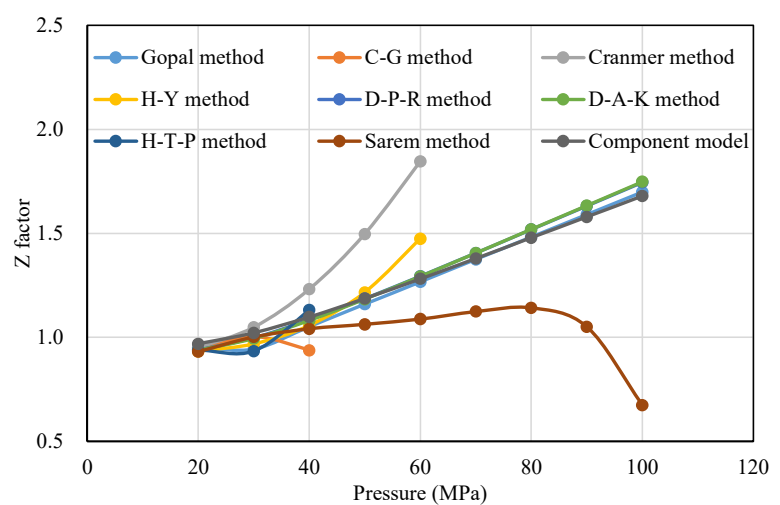

Fig4. Comparison of gas deviation factors $\left(146.7^{\circ} \mathrm{C}\right)$

As for the prediction of gas deviation factor of well flow, the component model has a high accuracy. Through the comparison with the prediction results of eight black oil model methods, the pressure has a great influence on the black oil model. When the pressure is lower than 40MPa, all the 7 black oil models except $\mathrm{Cr}$ method can accurately predict the gas deviation factor. However, when the pressure was higher than $40 \mathrm{MPa}$, only Gopal method and Dranchuk-abu-Kassem method remained basically consistent with the predicted results of the component model, and the deviation increased with the increase of the pressure. When the pressure is equal to $100 \mathrm{MPa}$, the deviation value between the predicted results of Gopal method and Dranchuk-abu-Kassem method and the component model is greater than 0.1 , and the prediction accuracy of gas deviation factor can no longer be guaranteed. In view of the above situation, it is suggested to use component model to predict the deviation factor of gas well flow.

\section{The influence of ultra-high pressure on the gas viscosity model}

The calculation of natural gas viscosity mainly includes Dempsey method, Lee method and Standing method. Through these three models, the influence of ultra-high pressure on the gas viscosity model of flow in ST1 is analyzed.

The fluid component data of ST1 were input into the PVT component model to calculate the gas viscosity of the well flow. By comparing the prediction results of three black oil model methods, Dempsey method, Lee method and Standing method, the influence of ultra-high pressure on the gas viscosity of the well flow was analyzed, as shown in FIG. 5 to 6 .

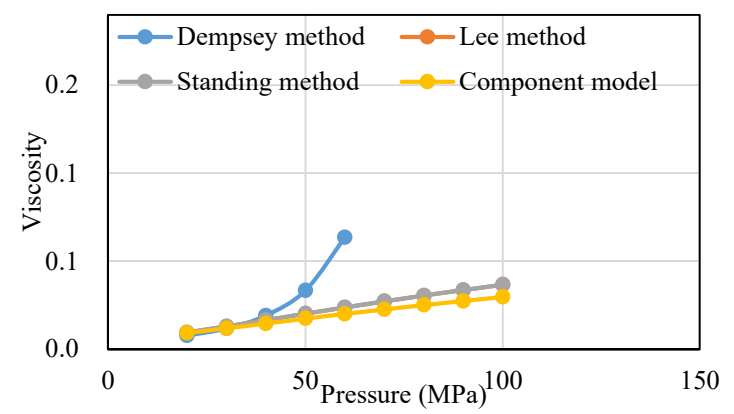

Fig5. Comparison of gas viscosity $\left(140^{\circ} \mathrm{C}\right)$

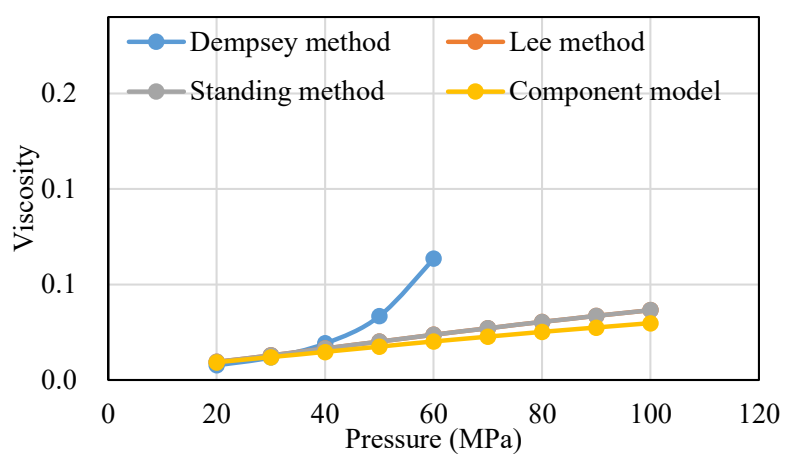

Fig6. Comparison of gas viscosity $\left(150^{\circ} \mathrm{C}\right)$

Combined with PVT phase analysis of gas reservoir fluid in double exploration well, the curve of gas viscosity changing with pressure in double exploration well 1 was compared with the model curve established in this paper (Figure 7, Figure 8). It can be seen from the comparison that the component model has high accuracy and can effectively predict the gas viscosity of ST1.

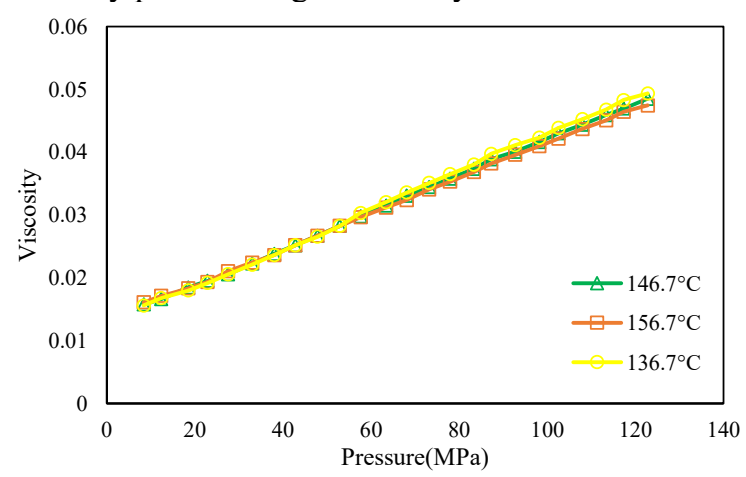

Fig7. Viscosity change curve with pressure (taken from The Natural Gas Reservoir Fluid Inspection Report of Double Exploration Well 1)

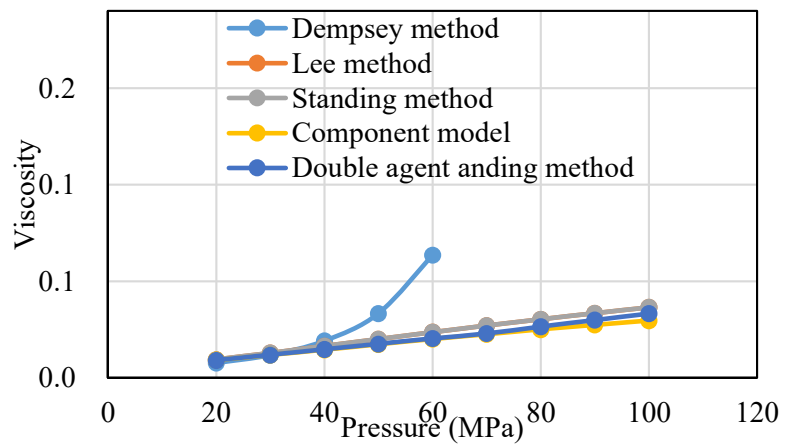

Fig8. Comparison of gas deviation factors $\left(146.7^{\circ} \mathrm{C}\right)$

As for the prediction of gas viscosity of well flow, the component model has a high precision. By comparing with the prediction results of three black oil model methods, the pressure has a great influence on the black oil model. When the pressure is lower than $40 \mathrm{MPa}$, the three black oil models can predict the gas viscosity more accurately. When the pressure is higher than $40 \mathrm{MPa}$, the Lee method and Standing method are basically consistent with the predicted results of the component model, and the deviation increases with the increase of the pressure. In the case that the pressure is equal to $100 \mathrm{MPa}$, the deviation value of the Lee method and the dual-detection method with the component model is greater than $0.01 \mathrm{cP}$, which 
cannot ensure the accuracy of gas deviation factor prediction. In view of the above situation, it is suggested to use component model to predict the viscosity of gas well flow.

\section{Conclusion}

(1) According to the research on wellbore pressure temperature prediction of ultra-high pressure gas Wells, the influence of ultra-high pressure on wellbore fluid physical property parameters cannot be ignored. The component model is adopted to calculate wellbore fluid PVT physical property, and the multi-phase flow model is modified to accurately predict wellbore pressure and temperature distribution.

(2) For the prediction of gas deviation factor of well flow, the component model has a relatively high accuracy. Through comparison with the prediction results of eight black oil model methods, the pressure has a greater impact on the black oil model. When the pressure is equal to $100 \mathrm{MPa}$, the deviation value between the predicted results of Gopal method and Dranchuk-Abu-Kassem method and the component model is greater than 0.1 , which cannot guarantee the prediction accuracy of gas deviation factor. It is suggested to use the component model to predict the deviation factor of gas well flow.

(3) For the prediction of gas viscosity of well flow, the component model has a relatively high accuracy. Through comparison with the prediction results of the three black oil model methods, the pressure has a greater impact on the black oil model. When the pressure is equal to 100 $\mathrm{MPa}$, Lee and double agent anding method and component model predicted results deviation is greater than $0.01 \mathrm{cP}$, is no guarantee that the gas deviation factor prediction accuracy, it is recommended to use component models to predict the viscosity of the gas well well flow content.

\section{References}

1. Zhang B J, LI X C, WANG X Z, DENG B, Xu Y H, Mi R X. (2017). Research and Application of ultrahigh pressure gas well test production Technology -A Case study of Well ST1 in north Sichuan region .Drilling and Production Technology, 59$61+86+55$.

2. Sun H F, LIU F, WANG Z M. (2017).Integrated Technology of high temperature and high pressure gas well test and completion .Drilling and Production Technology, 36-39+33.

3. Meng L Y, LI S L, MENG L H, NIU Y, Yang F L, LIU Y. (2016).Cause analysis of abnormal pressure test in high temperature and high pressure condensate well. Proceedings of the 2016 International Conference on Oil and Gas Field Exploration and Development, Beijing, China, 2016:6.

4. He J, Wei L L, GU Y, GUO X X, LEI W H. (2017). Productivity Evaluation of abnormal high pressure gas Wells under production conditions .Petrochemicals, 02-08.

5. $\mathrm{Xu} \mathrm{Y} \mathrm{D,} \mathrm{Gu} \mathrm{H} \mathrm{X.} \mathrm{(2017).} \mathrm{Interpretation} \mathrm{method} \mathrm{of}$ abnormal productivity data of high pressure and high yield gas Wells in carbonate rock [J].Journal of Southwest Petroleum University (Natural Science edition), 01-05.

6. Ai S, ZHANG T Y, XU Y D, DU J, PANG Wei. (2016).Productivity evaluation method of high temperature and high pressure gas Wells based on Constraint Optimization [J].Special Reservoirs, 7881+154-155

7. Liu H T, LI L L, WU J, LIU Y, HE Y. (2016). Optimal Configuration and Application of high temperature and high pressure gas well test string in front of Kuqa Mountain.Drilling and Production Technology, 4245+102-103

8. Xu Q X, WANG Y Z, JIANG Y C, ZHANG G, LI B, LIU Zeyu. (2017). Application of coiled tubing paraffin removal technology in high pressure oil and gas Wells [J].Petrochemical Applications, 83-85.

9. $\mathrm{Xu}$ Y F, ZHANG Z Q, FANG H L. (2017). Well control Technology for high temperature and high pressure gas Wells in Block $\mathrm{C}$ of the South China Sea.Oil Drilling Technology, 21-26. 